\title{
Targeting interleukin-17 via dopamine
}

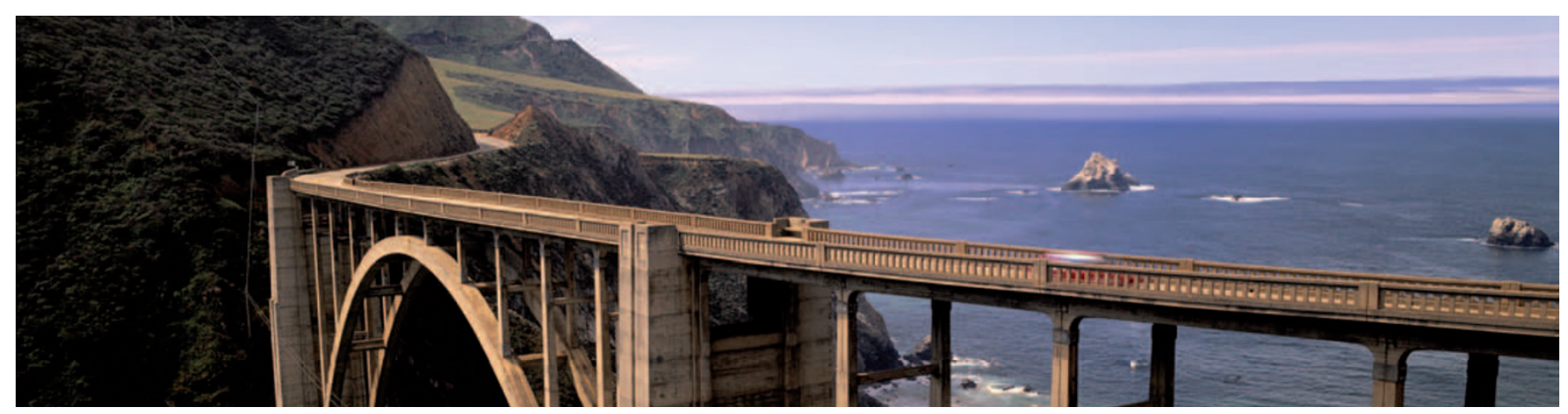

In addition to being a major neurotransmitter in the central nervous system, dopamine can contribute to the modulation of immunity via dopamine receptors expressed on immune cells. A recently published paper in the Journal of Immunology has shown that dopamine causes inflammation in rheumatoid arthritis by inducing the release of interleukin-17 (IL-17) from dendritic cells of the immune system. Moreover, a dopamine receptor antagonist suppressed rheumatoid arthritis pathology in a mouse model.

The study by Nakano and colleagues investigated the interaction between dopamine signalling and immune responses that are mediated by a specific subset of helper $\mathrm{T}\left(\mathrm{T}_{\mathrm{H}}\right)$ cells, $\mathrm{T}_{\mathrm{H}} 17$ cells, which produce cytokines including IL-17 and IL-21 and are involved in the pathogenesis of autoimmune and inflammatory disorders.

First, the authors studied human naive $\mathrm{CD} 4^{+} \mathrm{T}$ cells that were stimulated with CD3- and CD28-specific antibodies to expand and activate the $\mathrm{T}$ cell population. Dopamine increased IL-17 secretion from these cells, but only slightly increased IL-5 secretion, without affecting interferon- $\gamma$ production, which highlights that dopamine is important for the differentiation of naive $\mathrm{T}$ cells into $\mathrm{T}_{\mathrm{H}} 17$ cells rather than into $\mathrm{T}_{\mathrm{H}} 2$ cells. Pretreatment of $\mathrm{T}$ cells with an IL-6 receptor antibody inhibited IL-17 secretion, which suggests that dopamine increases IL-6-mediated IL-17 production. Furthermore, pretreatment of T cells with dopamine receptor 1 (D1)-like antagonists - SCH-23390 or LE300 — inhibited IL-17 secretion.

Next, to investigate the role of dopamine in the pathogenesis of rheumatoid arthritis, the authors measured catecholamine concentrations in synovial fluid taken from patients with the disorder. The concentration of dopamine (but not of noradrenaline or adrenaline) was higher in patients with rheumatoid arthritis than in control patients with osteoarthritis. Immunohistochemical studies showed the presence of dendritic cells in synovial tissue taken from patients with rheumatoid arthritis, which were stained positive for dopamine. Furthermore, accumulation of lymphocytes predominately occurred around dendritic cells. Together, these results suggest that dopamine- producing dendritic cells proliferate in the synovial tissue of patients with rheumatoid arthritis.

Finally, the authors studied a mouse model of arthritis in which severe combined immunodeficiency (SCID) mice were engrafted with synovial tissue and cartilage from patients with rheumatoid arthritis. Treatment of mice with D1-like antagonists (that were administered subcutaneously twice a week for 3 weeks) caused retraction of synovial tissue and shrinkage of synovial fibroblasts. In addition, mice that were treated with D1-like antagonists had minimal cartilage destruction compared to mice that were treated with D2-like antagonists.

The results of this study, together with similar positive results obtained using D1-like antagonists in mouse models of type 1 diabetes and multiple sclerosis, suggest that targeting G protein-coupled D1-like receptors could be an alternative to directly targeting IL-17 in inflammatory disease.

Charlotte Harrison

ORIGINAL RESEARCH PAPER Nakano, K. et al. Dopamine induces IL-6-dependent IL-17 production via D1-like receptor in $\mathrm{CD} 4$ naive T cells and D1-like receptor antagonist SCH-23390 inhibits cartilage destruction in a human rheumatoid arthritis/SCID mouse chimera model.J. Immunol. 186, 3745-3752 (2011) 